\title{
Using the Improvement of the Fixed Point Iterative Method in Solving Fredholm Integral Equations
}

Abbas Y. Al-Bayati

profabbasalbayati@yahoo.com

College of Computer Sciences and Mathematics

University of Mosul, Iraq

Received on: 04/08/2002

Accepted on: 07/09/2002

ABSTRACT

In this paper an iterative method for the fixed point is used to obtain an approximation solution for the Fredholm integral equations of the second kind by using several problems with different accuracy. Also we have proposed a successful improvement for the iterative fixed point method. A number of different tests are solved and compared in order to study the effectiveness of the proposed technique.

Keywords: fixed point method, Fredholm integral equations of the second kind.

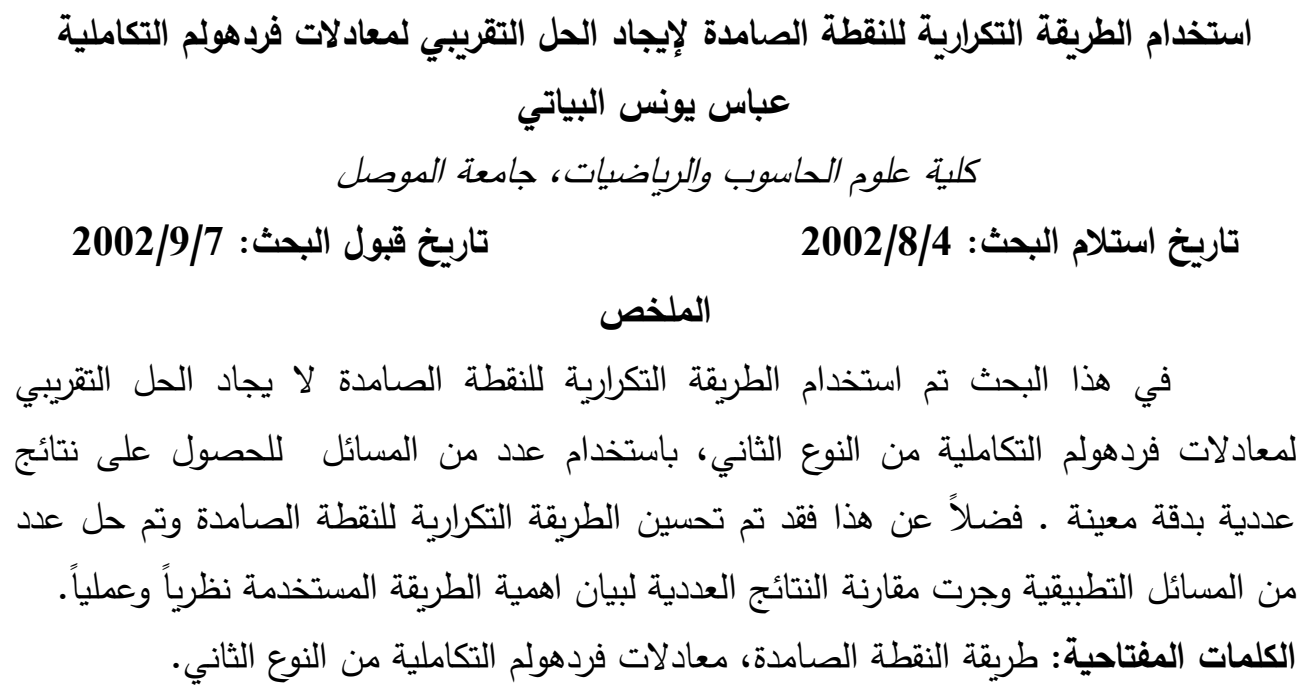

\section{1- Introduction:}

An equation, which contains an unknown function under the integral symbol, is called integral equation. A general example for general equation may be expressed as follows: Let

$$
\phi(\mathrm{s})=f(s)+\int K(x, t) u(t) d t
$$

Here $\mathrm{K}(\mathrm{x}, \mathrm{t})$ is a well-known function with two different variable and it is called the kernel of the integral equation; the function $f(x)$ is a known deriving term; and the function $\mathrm{u}(\mathrm{x})$ is un-known. (See for example Delves et al. 1985; Jerri, 1985; Athinso, 1997; Karashor et al. 1971).

The mathematical representation for a number of some special problems occur naturally by an integral equation. Some other problems, which are 
represented as differential equations with some conditions, may also be translated to an integral equation. There are some applications for the integral equation, such as "Electrostatic of anisotroic inclusions in anistropic media" by Helsing and Samulssan 1995; and "Flexural wave scattering in a conducting plate under a uniform magnetic field" by Shindo et al. 1997; also " Internal semi-infinite plane" by Goryaheva et al 1996; and "Solution of the two dimensional problem of earache in a uniform field in eddy- current nondestructive evaluation" by Harfield and Bowler 1995; and Abelis problems described in porter 1993.

To study the standard methods for the integral equations with one dimensional equation inside the Fredholm integral equation of the form:

$$
h(x) u(x)=f(x)+\lambda \int_{a}^{b} k(x, t) u(t) d t
$$

where $\mathrm{x}, \mathrm{t}$ defined on integral $[\mathrm{a}, \mathrm{b}]$ and $\mathrm{A}$ is parameter; when $\mathrm{h}(\mathrm{x})=1$, we obtain

$$
u(x)=f(x)+\lambda \int_{a}^{b} k(x, t) u(t) d t
$$

which is called Fredholm integral equation of the second kind while when $\mathrm{h}(\mathrm{x})=0$, we will obtain

$$
f(x)=\lambda \int_{a}^{b} k(x, t) u(t) d t
$$

this is called Fredholm integral equation of the first kind. In the eq.(3) and eq. (4) if the $\mathrm{k}(\mathrm{x}, \mathrm{t})=0$, when $\mathrm{t}>\mathrm{x}$ then

$$
u(x)=f(x)+\lambda \int_{a}^{x} k(x, t) u(t) d t
$$

and

$$
f(x)=\lambda \int_{a}^{x} k(x, t) u(t) d t
$$

These equations are called volterra equations of the second and first kinds. In eq.(2), when $f(x)=0$ this gives

$$
u(x)=\lambda \int_{a}^{x} k(x, t) u(t) d t
$$

this equation is called Homogeneous Fredholm equation. Also if in eq.(7), $\mathrm{k}(\mathrm{x}, \mathrm{t})=0$, at $\mathrm{t}>\mathrm{x}$ then

$$
u(x)=\lambda \int_{a}^{x} k(x, t) u(t) d t
$$


this equation is called Homogeneous volterra equation. All the forms eq.(3) to eq.(8) represent linear integral equations for unknown function $\mathrm{u}(\mathrm{x})$ :

\section{2- Modification for Iterative Formulation:}

The Fredholm integral equation of the second kind defined in eq.(3) can be written in a matrix form as follows:

$$
U=f+\lambda K U
$$

There are several numerical techniques used to solve eq.(3) successfully, one of these techniques is a numerical series technique which can be expressed as follows:

$$
U=\sum_{i=0}^{\infty} \lambda^{i} K^{i} f
$$

and as truncation formulation

$$
U \cong U_{n}=\sum_{i=0}^{n} \lambda^{i} K^{i} f
$$

if series defined in eq.(10a) converges then $\lim _{n \rightarrow \infty}\left|U-U_{n}\right|=0$, hence the approximation $\mathrm{U}_{\mathrm{n}}$ can be obtained by using the successive method, for which

$$
U_{n+1}=f+\lambda k U_{n}
$$

which the initial value $\mathrm{U}_{0}=\mathrm{f}$ the clearly notation of this function is defined by

$$
K U=\int_{a}^{b} K(x, t) U_{n}(t) d t
$$

Now for the improved iterative formula defined in eq.(11), suppose that

$$
U_{n+1}=M\left(U_{n}\right)
$$

And hence $M\left(U_{n}\right)=f+\lambda K U$. Now writing eq.(12)in a specific arrangement by adding $\beta u$ to both sided of eq.(12) with $\beta \neq-1$ gives $(1+\beta) u=\beta \mathrm{u}+M(u)$ this equation yields

$$
u=\frac{1}{1+\beta} N(u)+\frac{\beta}{1+\beta} u \cong M_{\beta}(u)
$$

If $\mathrm{u}^{*}$ satisfies eq.(12), then it must satisfy eq.(13) this means that $\mathrm{u}^{*}$ is a solution of eq.(11), hence the iterative formula

$$
u_{n+1}=M_{\beta}\left(u_{n}\right)
$$

Converges faster to the solution $u$ than the iterative formula are defined in eq.(11). Our choice for choosing the optimal value of the parameter $/ 3$ depends on the following formula

$$
\beta=1 / 2(D+G)
$$


where

$$
\begin{aligned}
& G=\inf _{[a, b]}|M(u)| \\
& D=\sup _{[a, b]}|M(u)|
\end{aligned}
$$

for more details of this specified choice see Davies 1987.

\section{3- Fixed Point and Contractive mapping:}

The very basic iterative method that we employed

$$
u_{n+1}=f(x)+\lambda \int k(x, t) u(t) d t
$$

was constructing solution, and the convergence of the sequence was constructing solutions, and the convergence of the sequence $u_{n}(x)$ to $u(x)$ has been studied for the original integral equation

$$
u(x)=f(x)+\lambda \int k(x, t) u(t) d t,
$$

Our treatment in this paper has been directed toward solving only linear integral equation as defined in eq.(18). The integral in eq.(18) is looked at in the following way, the right-hand side is considered as a mapping or transformation $\mathrm{T}$ on $\mathrm{u}$ denoted by $\mathrm{T}(\mathrm{u})$, while the left-hand side indicates that the transformation had left this one element $u$ unchanged.

$$
\mathrm{u}=\mathrm{T}(\mathrm{u})
$$

This means that the solution $\mathrm{u}$ which we seek for the integral eq.(18) represented a very special element in the domain of the operator $\mathrm{T}$. namely; that which remains unaltered or fixed under the $\mathrm{T}$ transformation. Such an element $\mathrm{u}$ as defined in eq.(19) is called a fixed point of the transformation (or mapping) $\mathrm{T}$. in this sense the successive approximation, solution of eq.(17) can be written as

$$
u_{n+1}=T\left(u_{n}\right) \quad n=0,1,2,3, \ldots
$$

\section{Definition (1):}

If a mapping $\mathrm{T}$ satisfies the Lipschitz condition on the interval $[\mathrm{a}, \mathrm{b}]$ then there exists a positive constant $\mathrm{L}$, such that

$$
|T(u)-T(v)| \leq|u-v| L
$$

for all values $u, v \in[a, b]$, where $[a, b]$ is the space of continuous functions closed interval $[\mathrm{a}, \mathrm{b}]$. The constant $\mathrm{L}$ is called Lipschitz constant.

\section{Definition (2):}

A map $\mathrm{T}$ is called contraction map on the closed interval [a,b], if it satisfies the following conditions:

$1-\mathrm{u} \mathrm{E}([\mathrm{a}, \mathrm{b}] \rightarrow \mathrm{T}(\mathrm{u}) \in([\mathrm{a}, \mathrm{b}]$.

This condition is called the closure condition. 
2- $\mathrm{T} \in \operatorname{Lip}[\mathrm{a}, \mathrm{b}]$ with Lipschitz constant, $0<\mathrm{L}<1$. For more details of these definitions see Davies 1987. Based on the above definitions: Let us consider the following theorem.

\section{Theory(1):}

If $\mathrm{T}$ is a contraction mapping on the interval $[\mathrm{a}, \mathrm{b}]$, then

a- The equation $\mathrm{u}=\mathrm{T}(\mathrm{u})$ has a unique solution $\mathrm{u}^{*} \mathrm{E}[\mathrm{a}, \mathrm{b}]$.

$\mathrm{b}$ - For any $\mathrm{u}_{\mathrm{o}} \in[\mathrm{a}, \mathrm{b}]$ the sequence defined in eq.(20) convergence to $\mathrm{u}^{*}$.

Proof: $\mathrm{T} \in \mathrm{Lip}[\mathrm{a}, \mathrm{b}]$ then $\mathrm{T} \in \mathrm{c}[\mathrm{a}, \mathrm{b}]$. Also since

$$
u=T(u)
$$

is continuous on the interval $[a, b]$, then we have to prove that it is a unique solution to eq.(22).On contrary, suppose that there exists another solution $\mathrm{v}^{*}$ such that $\mathrm{v}^{*} \neq \mathrm{u}^{*}$ which satisfy $\mathrm{u}^{*}=\mathrm{T}\left(\mathrm{u}^{*}\right), \mathrm{v}^{*}=\mathrm{T}\left(\mathrm{v}^{*}\right)$.

This implies that $|u *-v *|=\mid T\left(u *-T\left(v^{*}\right)|\leq| u *-v * \mid L\right.$; hence $L \geq 1$ which is a contradiction to the definition of the Lipschitz constant $(\mathrm{L} \leq 1)$. Since $\mathrm{T}$ is contraction mapping on the interval $[\mathrm{a}, \mathrm{b}] \rightarrow$, then $\mathrm{u}^{*}$ must be unique.

Now prove the second part (b) of the above theorem we employed the closure condition.

If $\mathrm{u}_{\mathrm{o}} \in[\mathrm{a}, \mathrm{b}]$, then $\mathrm{u}_{\mathrm{r}} \in[\mathrm{a}, \mathrm{b}]$; for $\mathrm{r}=1,2,3, \ldots$

$$
\left|\mathrm{u}_{\mathrm{r}-1}-\mathrm{v}^{*}\right|=\mid \mathrm{T}\left(\mathrm{u}_{\mathrm{r}-1}-\mathrm{T}\left(\mathrm{v}^{*}\right)|\leq| \mathrm{u}_{\mathrm{r}-1-\mathrm{v}^{*}} \mid \mathrm{L}\right. \text {; }
$$

so that we obtain

$$
\left|\mathrm{u}_{\mathrm{r}-1}-\mathrm{u}^{*}\right| \leq \mathrm{L}\left|\mathrm{u}_{0}-\mathrm{u}^{*}\right|
$$

since $0<\mathrm{L}<1$, then $\underset{t \rightarrow \infty}{L^{r}} \rightarrow 0$. This means $u_{r} \rightarrow u^{*}$ for $r \rightarrow \infty$.

This completes the proof of the above theorem.

Now we are going to use the same technique stated in section 2 to improve the fixed-point method. Start with eq.(19); add $\beta u$ to both sides of this equation with $\beta \neq-1$, to get

hence

$$
(1+\beta) \mathrm{u}=\beta \mathrm{u}+T(u),
$$

$$
\begin{gathered}
u=\frac{1}{1+\beta} T(u)+\frac{\beta}{\beta+1} u \cong T_{\beta}(u) \\
u^{*}=T\left(u^{*}\right) \square u^{*}=T_{\beta}\left(u^{*}\right) \\
\Rightarrow T_{R}\left(u^{*}\right)
\end{gathered}
$$

is a solution of eq.(3). (see Al-Husen, 1998).

Now the iterative formula

$$
u_{r+1}=T_{\chi}\left(u_{r}\right) ;(r=0,1,2 \ldots)
$$


Converges to the solution $\mathrm{u}$, faster than the iterative formula defined in eq.(20); this means that there exists an ideal value for the parameter $\beta$ which gives a faster convergence. Now calculating $\beta$ from eq.(15) and eq.(16) will vanish the process.

Now to study the effectiveness of the new procedure, let us consider the following example given in Al-Hassan (1998):

Example (1): Let $u(x)=1+\int_{0}^{1} x t^{2} u(t) d t$

By using the iterative method for the fixed point, the exact solution for the above problem is given by $u(x)=1+4 \mathrm{x} / 9$.

Now start with initial solution $\mathrm{u}_{\mathrm{o}}(\mathrm{x})=1$. By using iterative method for fixed point we obtain

$$
u(x)=1+\int_{0}^{1} x t^{2} u(t) d t=1+x / 3
$$

Similarly continue to get

$$
\begin{aligned}
& u_{2}(x)=1+5 \mathrm{x} / 12 \\
& u_{3}(x)=1+7 \mathrm{x} / 16 \\
& u_{4}(x)=1+85 \mathrm{x} / 192 \\
& u_{5}(x)=1+341 \mathrm{x} / 768 \\
& \vdots \quad: \quad \\
& \vdots \\
& u_{n}(x)=1+4 \mathrm{x} / 9, \text { which represent the exact solution. }
\end{aligned}
$$

The convergence condition in this problem is $|\lambda|<\sqrt{15}$. Again solving the above problem by using the improvement technique start with $\mathrm{u}_{\mathrm{o}}(\mathrm{x})=1$, by the iterative formula set

$$
u_{r+1}=\frac{1}{1+\beta}+\frac{\beta}{1+\beta} u_{r}(x)+\frac{1}{1+\beta} \int_{0}^{1} x t^{2} u r(t) d t \quad, \mathrm{r}=123 \ldots
$$

We have observed that the quantity of approximation solution will be obtained with one iteration at the value $(\beta=-0.25)$, at this value the quantity error equals zero. From eq.(15) and eq.(16) $\beta_{\text {opt }}=-1 / 6=-0.16667$ by using this value in the iterative procedure we get

$$
\begin{aligned}
& u_{1}(t)=1+2 \mathrm{x} / 5 \\
& u_{2}(x)=1+11 \mathrm{x} / 25 \\
& u_{3}(x)=1+111 \mathrm{x} / 250 \\
& u_{4}(x)=1+1111 \mathrm{x} / 2500 \\
& u_{5}(x)=1+11111 \mathrm{x} / 25000 \\
& \vdots \quad \quad \quad: \\
& \vdots \quad: \\
& u_{n}(x)=1+4 \mathrm{x} / 9
\end{aligned}
$$

Table (1) represents the convergence range of the sequence values which has been obtained by using $\beta_{o p t}=-1 / 6=-0.16667$ compared with the 
values which have been obtained by using $\beta=0.0$ (without improvement). The value of $\mathrm{e}_{n}$ has been calculated at the defined value for $\mathrm{x}=0.5$ the exact solution is given by $\mathrm{u}(0.5)=(1.222222)$.

Table (1)

Comparison between the error of exact and approximation solution at defined value for $x$ of example (1)

\begin{tabular}{|c|c|c|}
\hline $\mathbf{r}$ & $\beta=0.0$ & $\beta_{o p t} .=-0.16667$ \\
\hline & $\mathbf{e}_{\mathbf{n}}$ & $e_{\beta o p t .}$ \\
\hline 0 & 0.222222 & 0.222222 \\
\hline 1 & 0.055555 & 0.022222 \\
\hline 2 & 0.13889 & $2.2222 \times 10^{-3}$ \\
\hline 3 & $3.4722 \times 10^{-3}$ & $2.222 \times 10^{-4}$ \\
\hline 4 & $8.68 \times 10^{-4}$ & $2.22 \times 10^{-5}$ \\
\hline 5 & $2.17 \times 10^{-4}$ & $2.2 \times 10^{-6}$ \\
\hline 6 & $5.42 \times 10^{-5}$ & $2.0 \times 10^{-6}$ \\
\hline 7 & $1.35 \times 10^{-5}$ & 0.0 \\
\hline
\end{tabular}

Clearly from table (1) the new proposed technique is most efficient and its value of convergence is quadratic and it saves few iteration in obtaining the exact solution when compared with the standard fixed method with out loosing any extra evaluations.

\section{4- An Accelerated Iterative Method:}

To accelerate the modified technique discussed in Al-Husen and AlHussan (1998), we may write the kernel equation

$$
k_{i}(s, t)=\int_{0}^{s} k(s, z) k_{i-1}(z, t) d z
$$

In the same manner adding $\beta \mathrm{k}(\mathrm{s}, \mathrm{t})$ for the both sides of the above equation

$$
k(s, t)+\beta k(s, t)=\int_{0}^{s} k(s, z) k(z, t) d z+\beta k(s, t) . \quad \text { with } \beta \neq-1
$$

This may be reduced to

$$
(1+\beta) k_{\beta}(s, t)=\beta k(s, t)+\int_{0}^{s} k(s, z) k(z, t) d z
$$

so that

$$
k_{\beta}(s, t)=\frac{\beta}{1+\beta} k_{\beta}(s, t)+\frac{1}{1+\beta} \int_{0}^{s} k(s, z) k(z, t) d z
$$

and hence the new proposed iterative formula becomes: 


$$
k_{\beta}(s, t)=\frac{\beta}{1+\beta} k_{\beta_{i-1}}(s, t)+\frac{1}{1+\beta} \int_{0}^{s} k(s, z) k_{\beta_{i-1}}(z, t) d z \quad \mathrm{i}=2,3, \ldots
$$

Calculating 8 by formula (15) or (16) gives an effective numerical results.

Example (2) : Let $\phi(s)=f(s)+\lambda \int_{0}^{s} s t \phi(t) d t$

\section{Solution:}

$$
\begin{aligned}
k_{l}(s, t) & =k(s, t)=s t \\
\Rightarrow k_{2}(s, t) & =\frac{1}{3} s t \\
\Rightarrow k_{3}(s, t) & =\left(\frac{1}{3}\right)^{2} s t \\
\vdots & \\
\Rightarrow k_{n}(s, t) & =\left(\frac{1}{3}\right)^{n-1} s t
\end{aligned}
$$

The exact solution of this problem is

$$
\phi(\mathrm{s})=1.25
$$

$\beta_{\text {opt. }}$ can be calculated from eqs.(15), (16), so that $\beta_{\text {opt. }}=-0.125$.

Using the value of $\beta_{\text {opt. }}$ in the new proposed iterative eq.(25) yields:

$$
\begin{aligned}
& k_{\beta_{1}}(s, t)=s t \\
& k_{\beta_{2}}(s, t)=\left(\frac{5}{21}\right) s t \\
& k_{\beta 3}(s, t)=\left(\frac{5}{21}\right)^{2} s t \\
& \cdots \\
& k_{\beta n}(s, t)=\left(\frac{5}{21}\right)^{n} s t
\end{aligned}
$$

The approximated value of $\phi_{\beta}(\mathrm{s})$ can be calculated from

$$
\phi(s)=s+\frac{3}{5} \int_{0}^{s} s t^{2} d t
$$

so that

$$
\phi \beta(\mathrm{s})=1.195
$$

which is very close to the exact value of the integral.

\section{5- Conclusion:}

We have modified an iterative method for fixed point iteration to obtain an approximate solution for Fredholm integral equation of the $2^{\text {nd }}$ kind. Also we have developed an accelerated version of the method. 


\section{REFERENCES}

[1] Al-Hassan,M. (1998) ." Numerical solution of Fredholm Integral Equations of the $2^{\text {nd }}$ kind" Msc.Thesis, Mosul University, Iraq.

[2] Atkinson, K., (1997), "The numerical solution of integral equation of the $2^{\text {"d }}$ kind, Cambridge university press, (552) pages.

[3] Davies, A.R. (1987), "Lectures in numerical analysis (unpublished), the University College of Wales Aberyrtwyth.

[4] Delves, L.M. and Mohammed, L., (1985), " Computational method for integral equations", Cambridge's University Press, Cambridge.

[5] Goryacheva, I. ; Sadeghi, F. ; Nickel, D.A., (1996), " Internal stresses in contact of a Rough Body and a viscoelastic Layered semiinfinite plane, Journal of Tribology, Vol. 118, January, page (131-136).

[6] Harfield, N.; Bowler, J.R. (1995), "Solution of the twodimensional problem of a crack in a uniform field in addy- current nondestructive evaluations Journal phys.D: Appl. Phys. 28, pp. (21972205).

[7] Helsing, J.; Samudsson, K., (1995), Electrostatics of anisotropic inclusions in anisotropic Media, Journal, phys., Vol. 78, No.4,15 August, pp. (2498-2503).

[8] Jerri, A.J., (1985), "Introduction to integral equations with application", Morcel Dekker, Inc. Newyork.

[9] Krasnov, M.; Kiselev, A.; Makarenko, G., (1997) "Problems and exercise in integral equations", (English Translation), Mir Publisheres, Moscow.

[10] Porter, D.; Stirling, D.S.G., (1993), "Integral equations practical treatment from spectral theory to applications", Cambridge University Press, Cambridge.

[11] Shindo, Y.; Ohnishi, L; Tohyama, S., (1997), "Flexural were scattering at a through crack in a conducting plate under a uniform magnetic field, Journal of Applied Mechanics, Vol. 64, pp. (828834). 